Bangladesh J. Bot. 49(2): 349-355, 2020 (June)

\title{
MODELING METHOD OF STATIC CONTACT ANGLE OF DROPS ON LEAF SURFACE OF THE TYPICAL CROPS
}

\author{
Wang Shujie, Li Tong, Li Xiaohui, Bai He and Zhou Yajun ${ }^{1 *}$ \\ College of Biological and Agricultural Engineering, Jilin University, \\ Changchun-130022, China
}

Keywords: Surface wettability, Spreading features, Contact angle, MATLAB, Pesticide droplets

\begin{abstract}
It was found that the pesticide droplets have always showed an elliptical shape when detecting the contact angle of the pesticide droplets on the Ken-Nian No. 1 corn leaves. In order to describe more accurately the spreading behavior of the pesticide droplets on these corn leaves, present authors have established a contact angle prediction model. In this experiment, the leaves in Ken-Nian No. 1 corn at jointing stage were used as test materials and were sprayed with different concentrations of Kresoxim-methyl water dispersant pesticide. The simulation test has used the modified HAD-HB contact angle tester to measure the four variates, longitudinal and horizontal spreading diameter named ' $a$ ' and ' $b$ ', longitudinal and lateral contact angle named ' $\alpha$ ' and ' $\beta$ '. The mathematical relationship models between ' $\alpha$-ab' and ' $\beta$-ab' were established by using Matlab. The Adjusted R-square of two models are above 0.98. The test results showed that the predicted values of the models were within \pm 2 degrees of the actual measured value.
\end{abstract}

\section{Introduction}

Contact angle is an important parameter of wetting degree, which directly reflects the hydrophilic and hydrophobic properties of the solid surface (Roach et al. 2008). In the agricultural sector, wetting degree will directly affect the deposition of pesticides on the surface of crops.

The most classical models of contact angles are Young's equation, Wenzel equation and Cassie equation (Young 1800, Wenzel 1936, Cassie 1948). Using a model that predicts contact angle as a function of roughness, the roughness factor for the hydrophobic leaves has been calculated, which is used to calculate the contact angle for a flat leaf surface (Zachary et al. 2006). The hydrophobic models of papilla-ciliary and fold-setal non-smooth surfaces were set up to determine the impacts of geometric parameters on the hydrophobicity (Ren et al. 2007). A model for predicting the contact angles of water and oil droplets was proposed by Yong et al. (2009). Chu et al. (2017) investigated that the interaction between droplet growth and surface contact angle. Jiang et al. (2018) built the AR-DCA model based on Huffman function to better simulate the droplet deformation and evolvement. In existing research, dynamic contact angle models are more than static contact angle models (Jiang et al. 1979, Seebergh et al. 1992).

On non-smooth surfaces, droplets are affected by surface tension, surface free energy and surface roughness during wetting and spreading (Neinhuis and Barthlott 1998, Holder 2007). Actually it is difficult to spread evenly around. Therefore, the contact angle in a single direction is not sufficient to explain the spreading and wetting behavior. Based on the current method of measuring contact angle (Edward 1983, Bunster et al. 1989, Pinon et al. 2006, Guo et al. 2007), in order to describe more fully the wetting and spreading properties of the droplets, the authors have tried to establish a model for predicting contact angles in different directions. That is to establish a mathematical relationship model of ' $a$ ', ' $b$ ', ' $\alpha$ ' and ' $\beta$ '.

*Author for correspondence: <1057265937@qq.com>. ${ }^{1}$ College of Food Science and Engineering, Jilin University, Changchun, China 130062. 


\section{Materials and Methods}

The characteristic leaves in the Ken-Nian No. 1 corn at jointing stage, 60\% Kresoxim-methyl water dispersant pesticide were used for the experiment. These experiments were conducted in the glasshouse (4351'05”N, 12519'51'E), which located in the College of Biological and Agricultural Engineering of Jilin University. The temperature of glasshouse is controlled at 24 \pm $4^{\circ} \mathrm{C}$ in the daytime, and at $17 \pm 2^{\circ} \mathrm{C}$ at night.

The modified HAD-HB contact angle tester was used. The $60 \%$ Kresoxim-methyl water dispersant was diluted into different concentrations solution for use. The concentrations of pesticide were $0.33,05,0.6,0.716,0.8,1$ and $2 \mathrm{~g} / \mathrm{l}$. After washed corn leaves were dried naturally. The sample should be free from damage and the sample position should be away from the veins. Then the samples was fixed on the contact angle measuring instrument. Then pictures from three angles (up, left, front) were taken. A new sample was replaced for each test which was repeated three times.

The surface of corn leaves has a long strip of grain, which causes the spread of droplets on the leaves to appear as part of the ellipsoid. It was designated as the length of the droplet spread along the direction of the vein. The authors designated ' $a$ ', as the longitudinal spreading diameter, the length of the perpendicular direction, and 'b', as the horizontal spreading diameter, the contact angle measured when the line of sight of the camera is perpendicular to the direction of the vein, ' $\alpha$ ' was designated as the longitudinal contact angle, and ' $\beta$ ' was designated as the parallel direction the lateral contact angle.

Regarding the basic theory of the modeling part in this experiment, the small droplet (spherical crown) method is adopted because of the simple operation and high precision. The height (h) and width (2r) of small droplets were measured on a solid plane to get the contact angle, according to:

$$
\tan \frac{\theta}{2}=\frac{h}{r}
$$

By observing the spreading pattern of droplets on corn leaves, it can be approximated as part of an ellipsoidal shape, which can still be regarded as a spherical crown by two-dimensional projection in either direction. Therefore, the method of measuring the contact angle by the small droplet (spherical crown) method is still feasible here.

Since the droplet volume is constant for each test by using micro syringe, there is a functional relationship between ' $h$ ' and ' $r$ ' of the formed spherical crown droplets (Lu et al. 2013). The contact angle $\theta$ can be obtained by the height (h) and width (2r) of the small droplet by the formula (1). Similarly, when the droplets exhibited an ellipsoidal shape, the longitudinal spreading diameter ' $a$ ' and the horizontal spreading diameter ' $b$ ' also have a functional relationship with the contact angles of the two directions. According to this, the contact angle model is established.

\section{Results and Discussion}

The contact angle of the droplet completely spread on the surface of the corn leaf has been measured and is presented in Fig. 1. It can be seen from the figure that the both longitudinal and lateral contact angles followed asimilar pattern i.e. decreases first and then increases slowly with the increase of the concentration of the pesticide. The contact angle is minimal at concentrations around $0.716 \mathrm{~g} / \mathrm{l}$. The longitudinal contact angle was $39.1^{\circ}$ and the lateral contact angle was $42.3^{\circ}$. The longitudinal contact angles of the droplets are all smaller than the lateral contact angle. This indicates that the droplets tend to spread along the leaf vein direction on the leaf surface. 
Through the test, the relationship between the average spreading diameter and the concentration was measured (Fig. 2). It can be seen from the figure that as the concentration increases, the spreading diameter has increased first and then decreased slowly. When the concentration reached around $0.716 \mathrm{~g} / \mathrm{l}$, the spreading diameter has reached the maximum in both cases. The largest longitudinal spreading diameter was $3910.8 \mu \mathrm{m}$, and the largest horizontal spreading diameter was $3601.2 \mu \mathrm{m}$. The longitudinal spreading diameter was larger than the horizontal spreading diameter, indicating that the droplets tend to spread along the vein direction on the leaf surface.

The relationship between the height of the droplets and concentration was measured in this experiments (Fig. 3). It is apparent that as the concentration increases, the droplet height has firstly decreased and then increased. At a concentration of around $0.716 \mathrm{~g} / \mathrm{l}$, the droplet height reached the minimum and it was $718.7 \mu \mathrm{m}$.

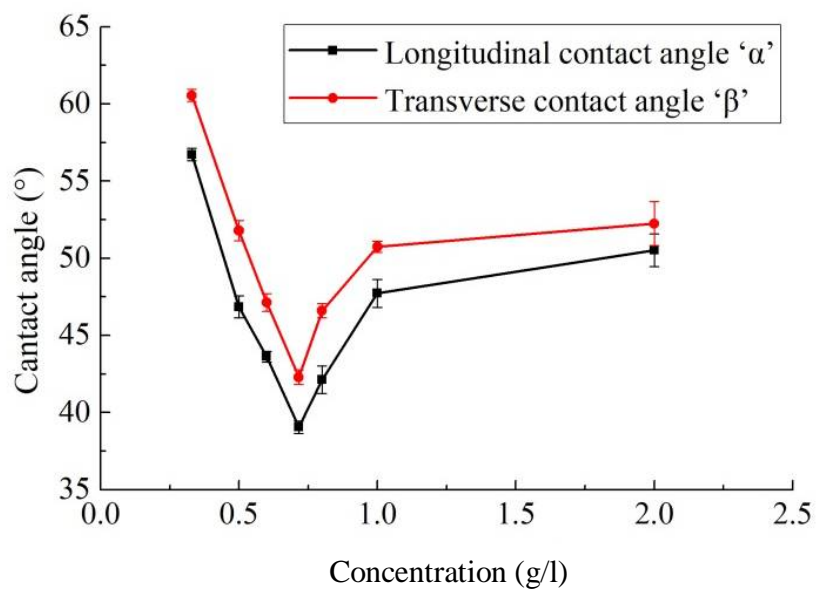

Fig. 1. Relationship of ' $\alpha$ ', ' $\beta$ ' and concentration.

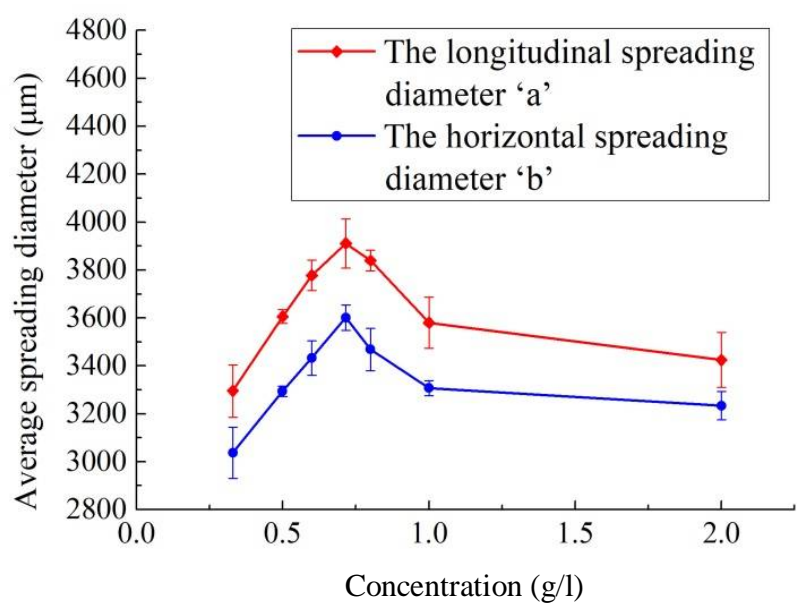

Fig. 2. Relationship of 'a', 'b' and concentration. 


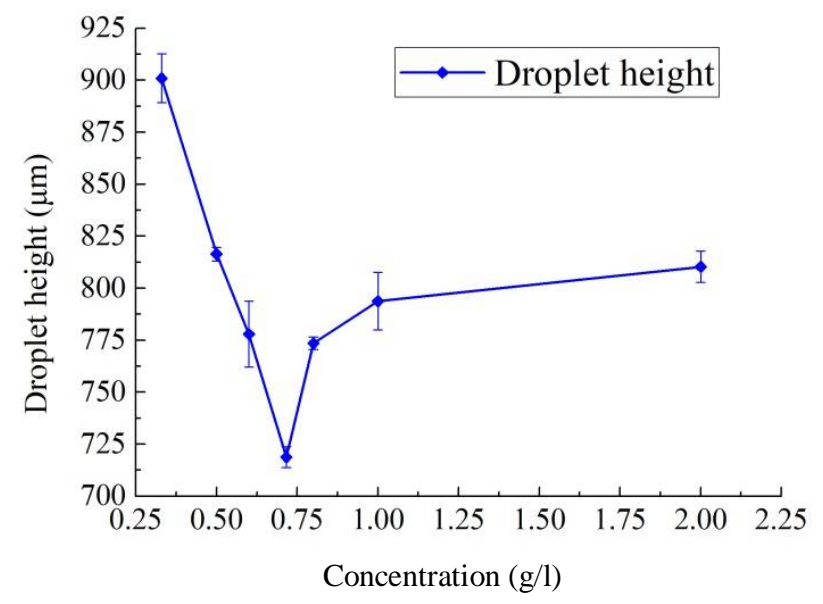

Fig. 3. Relationship between droplet height and concentration.

The change in contact angle caused by the difference in concentrations was observed. This can be seen as the interaction among the height of the droplet, the longitudinal spreading diameter and the horizontal spreading diameter. Therefore, the relationship model between the contact angle and the spread diameter was established by the above three variables.

The longitudinal spreading diameter ' $a$ ' and the horizontal spreading diameter ' $b$ ' were used as independent variables, and the longitudinal contact angle ' $\alpha$ ' was used as the dependent variable. The mathematical function relationship of the curved surface was established by using the MATLAB curve fitting function (Fig. 4).

The equation for the relationship of ' $\alpha$ ', ' $a$ ' and ' $b$ ' are as follows:

$$
\alpha=274.7-0.1035 \mathrm{x} 1+0.005983 \mathrm{x}_{2}+2.823 \times 10^{-5} \mathrm{x}_{1} \mathrm{x}_{2}-1.928 \times 10^{-5} \mathrm{x}_{2}^{2}
$$

The sum of squares due to error (SSE) of the equation was 0.8568, the R-square was 0.9958, the adjusted R-square was 0.9875 , and the root mean squared error (RMSE) was 0.6545 . The R-square of the model showed that there was $99.58 \%$ probability that the data had fitted the model.

The longitudinal spreading diameter ' $a$ ' and the horizontal spreading diameter ' $b$ ' were used as independent variables, and the lateral contact angle ' $\beta$ ' was used as the dependent variable. The mathematical function relationship of the curved surface was established by using the MATLAB curve fitting function (Fig. 5).

The equation for the relationship of ' $\beta$ ', ' $a$ ' and ' $b$ ' is shown as follows:

$$
\beta=234.2-0.09654 \mathrm{x}_{1}+0.02678 \mathrm{x}_{2}-1.32 \times 10^{-4} \mathrm{x}_{1}^{2}+3.179 \times 10^{-4} \mathrm{x}_{1} \mathrm{x}_{2}-1.827 \times 10^{-4} \mathrm{x}_{2}^{2}
$$

The SSE of the equation was 0.2183 , the R-square was 0.9989 , the adjusted R-square was 0.9934 , and the RMSE was 0.4672 . The R-square of model showed that there was $99.89 \%$ probability that the data fitted the model. 


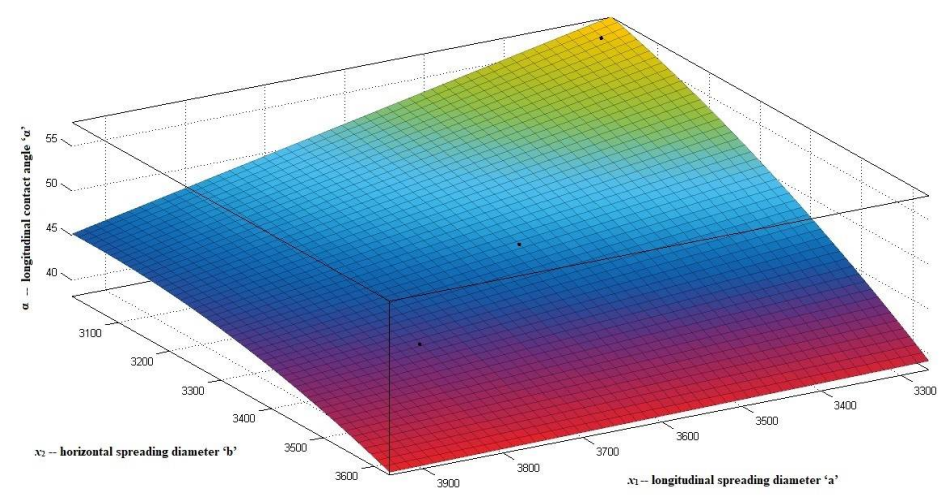

Fig. 4. Model of relationship between longitudinal contact angle ' $\alpha$ ' and longitudinal, horizontal spreading diameter ' $a$ ' and ' $b$ '.

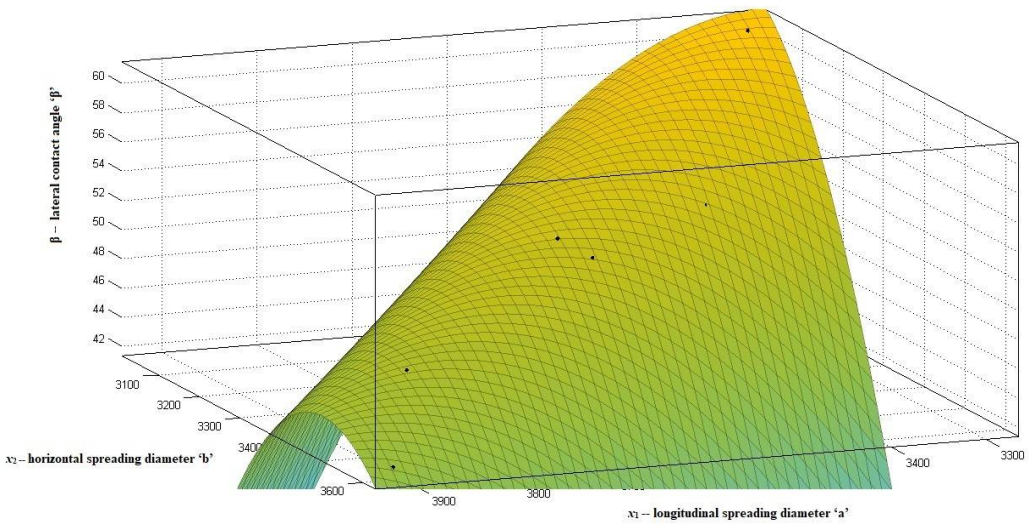

Fig. 5. Model of relationship between the lateral contact angle ' $\beta$ ' and longitudinal, horizontal spreading diameter ' $a$ ' and ' $b$ '.

To verify the reliability of the model, a set of verification tests was performed. Three methods were used to measure the contact angle, as the contact angle measurement tester, the $\theta / 2$ method, and the model calculation. Based on the measured value, $\pm 2^{\circ}$ was the error. The accuracy of the contact angle was obtained by comparing the three methods. The results were shown in Figs 6 and 7.

From Figs 6 and 7, the contact angle value calculated by the $\theta / 2$ method and models were within the error line range with $\pm 2^{\circ}$ as the error line range based on the measured value. In this way, the reliability of this model was verified.

A new modeling idea for measuring longitudinal and lateral contact angles has been proposed. And the model about the relationship of ' $\alpha$ ', 'a' and ' $b$ ' has been established. It was : $\alpha=274.7-$ $0.1035 \times$ x $1+0.005983 \times \times 2+2.823 \times 10-5 \times \times 1 \times \times 2-1.928 \times 10-5 \times \times 22$. The model about the relationship of ' $\beta$ ', ' $a$ ' and ' $b$ ' has been established. It was:

$\beta=234.2-0.09654 \times x 1+0.02678 \times \times 2-1.32 \times 10-4 \times x 12+3.179 \times 10-4 \times \times 1 \times \times 2 \times-1.827 \times 10-4 \times \times 22$. 


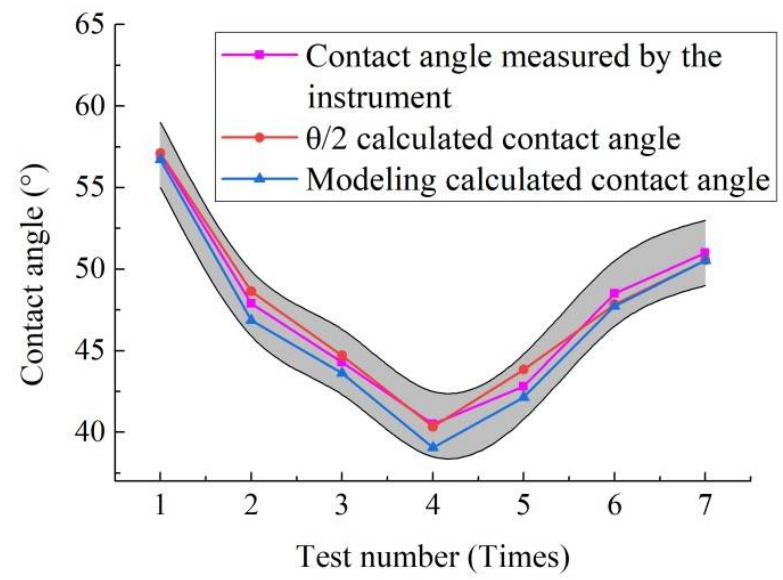

Fig. 6. Longitudinal contact angle under three methods.

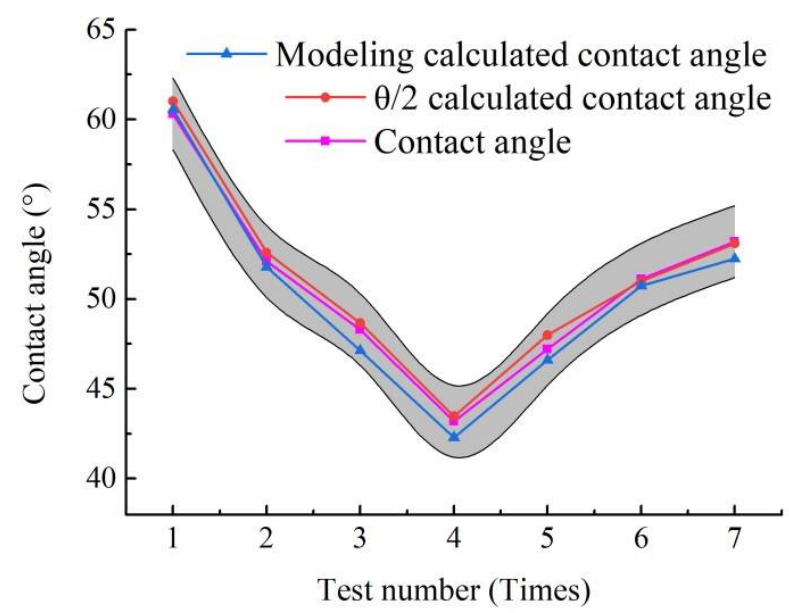

Fig. 7. Lateral contact angle under three methods.

At the same time, the accuracy of the model was verified by experiments, and the calculated values were within the allowable error range of the measured values. The predicted value of the model was close to the real value, and the deviation was small, it proved the feasibility and effectiveness of the static contact angle modeling method proposed in this paper. At present, most of the research on the contact angle model is to fit the outer curve of the droplet, or to predict the single contact angle with different conditions ( $\mathrm{Xu}$ and $\mathrm{Lu}$ 2013). The model for predicting the longitudinal and lateral contact angles proposed in this paper can more accurately predict and describe the wetting and spreading behavior of droplets on the leaf surface. By confirming the behavior of pesticide droplets, one can reduce the loss of pesticide, increase the amount of deposition, reduce environmental pollution and protect the environment. Simultaneously, this new model provided the basis for the establishment of a multi static contact angle database for a variety of monocot leaves. 


\section{Acknowledgments}

This work was supported by the Jilin Province science and technology development key program (No. 20180201009NY), Key Laboratory of Ministry of Education (No. K201101) and Project 2017121 Graduate Innovation Fund of Jilin University. The authors thank of the authorities the School of Biological and Agricultural Engineering at Jilin University and Key Laboratory of Ministry of Education of engineering bionics for support.

\section{References}

Bunster L, Fokkema NJ and Schippers B 1989. Effect of surface-active Pseudomonas spp. on Leaf Wettability. Appl. Environ. Microb. 55: 1340-1345.

Cassie ABD 1948. Contact angles. Discussions of the Faraday Society 3: 11-16.

Chu FQ, Wu XM and Ma Q 2017. Condensed droplet growth on surfaces with various wettability. Appl. Therm. Eng. 115: 1101-1108.

Edward LS 1983. Electrostatic pesticide spraying: Concepts and Practice. IEEE T. Ind. Appl. IA-19: 160-168.

Guo ZG and Liu WM 2007. Biomimic from the superhydrophobic plant leaves in nature: Binary structure and unitary structure. Plant. Sci. 172(6): 1103-1112.

Holder CD 2007. Leaf water repellency of species in Guatemala and Colorado (USA) and its significance to forest hydrology studies. J. Hydrol. 336: 147-154.

Jiang MC, Zhou B and Wang XC 2018. Comparisons and validations of contact angle models. Int. J. Hydrogen Energ. 43(12): 6364-6378.

Jiang TS, Oh SG and Slattery JC 1979. Correlation for dynamic contact angle. J. Colloid Interf. Sci. 69: 74-77.

Lu J, Zhang HT, Wei DY and Hu YX 2013. Dynamic contact angle forecasting and modeling for drop-leaf interface based on time series analysis. Transactions of the Chinese Society for Agricultural Machinery. 44(02): 80-86.

Neinhuis C and Barthlott W 1998. Seasonal changes of leaf surface contamination in beech, oak, and ginkgo in relation to leaf micromorphology and wettability. New Phytol. 138(1): 91-98.

Pinon J, Frey P and Husson C 2006. Wettability of poplar leaves influences dew formation and infection by Melampsora larici-populina. Plant Dis. 90(2): 177-184.

Ren LQ, Wang SJ, Tian XM, Han ZW, Yan LN and Qiu ZM 2007. Non-smooth morphologies of typical Plant leaf surfaces and their anti-adhesion effects. J. Bionic Eng. 4(1): 33-40.

Roach P, Shirtcliffe NJ and Newton MI 2008. Progess in super-hydrophobic surface development. Soft Matter. 4(2): 224-240.

Seebergh JE and Berg JC 1992. Dynamic wetting in the low capillary number regime. Chem. Eng. Sci. 47: 4455-4464.

Wenzel RN 1936. Resistance of solid surfaces to wetting by water. Industrial and Engineering Chemistry. 28: 988-994.

Xu ZN and Lu FC 2013. A static contact angle algorithm and its application to hydrophobicity measurement in silicone rubber corona aging test. IEEE T. Dielect. El. In. 20(5): 1820-1831.

Yong CJ and Bharat B 2009. Wetting behavior of water and oil droplets in three-phase interfaces for hydrophobicity/philicity and oleophobicity/philicity. Langmuir. 25(24): 14165-14173.

Young T 1800. An essay on the cohesion of fluids. Proceedings of the Royal Society of London. 1: 171-172.

Zachary B and Bharat B 2006. Surface characterization and adhesion and friction properties of hydrophobic leaf surfaces. Ultramicroscopy 106: 709-719.

(Manuscript received on 12 October, 2018; revised on 25 March, 2019) 